\title{
Spanning Maximal Planar Subgraphs of Random Graphs
}

\section{B. Bollobás*}

Department of Mathematics, Louisiana State University, Baton Rouge, LA 70803

\author{
A. M. Frieze $\dagger$ \\ Department of Mathematics, Carnegie-Mellon University, Pittsburgh, PA 15213
}

\begin{abstract}
We study the threshold for the existence of a spanning maximal planar subgraph in the random graph $G_{n, p}$. We show that it is very near $p=\frac{1}{n^{1 / 3}}$. We also discuss the threshold for the existence of a spanning maximal outerplanar subgraph. This is very near $p=\frac{1}{n^{1 / 2}}$.
\end{abstract}

Key Words: random graphs, planar graphs.

\section{INTRODUCTION}

In this short note we condider the threshold for the property that the random graph $G_{n, p}$ contains a spanning maximal planar subgraph, i.e., a planar subgraph with $3 n-6$ edges and $2 n-4$ triangular faces. Our notation and terminology follows [1]; in particular $G_{n, p}$ is the random graph with vertex set $[n]=$ $\{1,2, \ldots, n\}$ which is obtained by selecting each of the $N=\left(\begin{array}{l}n \\ 2\end{array}\right)$ possible edges independently, with probability $p$. Let us define the graph property $\mathscr{A}$ by setting $G=(V, E) \in \mathscr{A}$ if $E$ contains a set $F$ of $3|V|-6$ edges such that $(V, F)$ is planar. Thus $G \in \mathscr{A}$ iff $G$ contains a maximal planar graph spanning the entire graph.

${ }^{*}$ Research supported in part by NSF Grant DMS-8806097.

$\dagger$ Research supported in part by NSF Grant CCR-8900112.

Random Structures and Algorithms, Vol. 2, No. 2 (1991)

(C) 1991 John Wiley \& Sons, Inc. CCC 1042-9832/91/020225-07\$04.00 


\section{Theorem 1.}

(a) Let $p=\frac{c}{n^{1 / 3}}$ where $c=(27 e / 256)^{1 / 3}$. Then a.e. $G_{n, p} \notin \mathscr{A}$.

(b) Let $p=\frac{c(\log n)^{1 / 3}}{n^{1 / 3}}$ where $c \geq 100$. Then a.e. $G_{n, p} \in \mathscr{A}$.

Note the small gap within which the exact threshold has been located. It is difficult to speculate what the exact threshold value is. Note also that the simplest "local" condition that every vertex lies on at least one triangle is not almost always sufficient, in contrast with many other graphs properties (see [1]). Note also that $n^{-k /(3 k-6)}$ is the exact threshold for containing any fixed maximal planar subgraph with $k$ vertices. The techniques used to prove Theorem 1 can be modified to prove another problem.

Recall that a graph is outerplanar if it can be drawn on the plane with every vertex incident with the outer face. A maximal outerplanar graph is one in which every face other then the outer one is a triangle. An $n$-vertex maximal outerplanar graph has $2 n-3$ edges.

Let $\mathscr{B}$ denote the property of containing a maximal outerplanar graph spanning the entire graph.

\section{Theorem 2.}

(a) Let $p=\frac{c}{n^{1 / 3}}$ where $c=(e / 4)^{1 / 2}$. Then a.e. $G_{n, p} \notin \mathscr{B}$.

(b) Let $p=\frac{c(\log n)^{1 / 2}}{n^{1 / 2}}$ where $c>8 \sqrt{2}$. Then a.e. $G_{n, p} \in \mathscr{B}$.

The proofs of these two theorems are given in the next two sections.

\section{PROOF OF THEOREM 1}

Let $M_{n}$ be the number of maximal planar subgraphs with $n$ labelled vertices. Then

$$
P\left(G_{n, p} \in \mathscr{A}\right) \leq M_{n} p^{3 n-6}
$$

As almost every maximal planar graph has a trivial automorphism group, Tutte's classical formula [4] implies that

$$
M_{n} \leq n^{n-3}\left(\frac{256}{27}\right)^{n}
$$

if $n$ is sufficiently large (in fact, for all $n$ ). Hence, if $p=\frac{c}{n^{1 / 3}}$ where $c=(27 e / 256)^{1 / 3}$, then

$$
P\left(G_{n, p} \in \mathscr{A}\right)<1 / n
$$

and this proves part (a). 


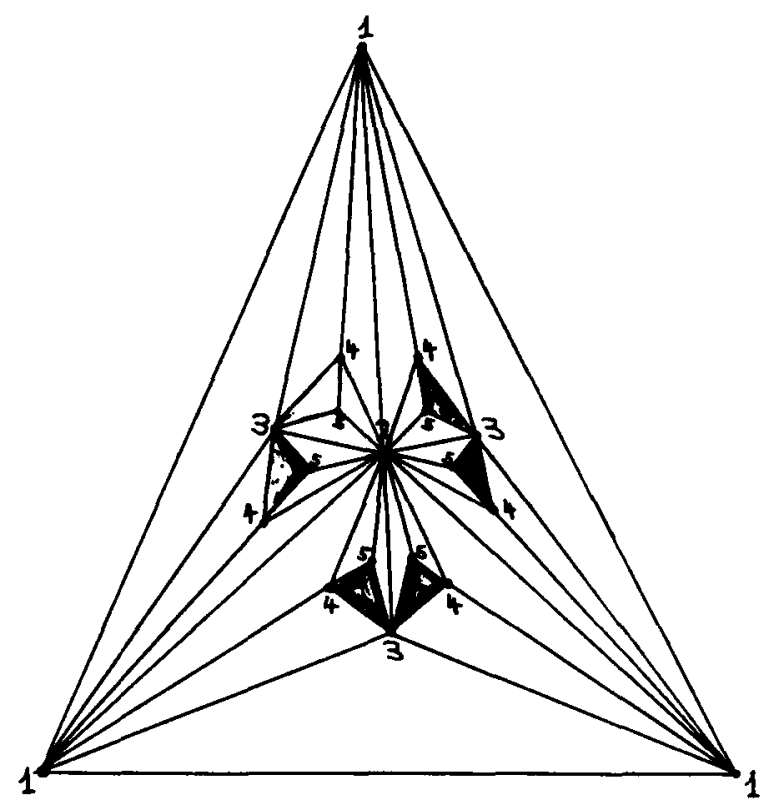

Fig. 1

To prove part (b) we need to define some specific triangulations that we can construct with high probability. Let $T=T_{1}$ be the 19-vertex triangulation of Figure 1. Note that $T_{1}$ can be constructed from the outer triangle by a sequence of "vertex insertions." By this we mean take a face $F=x y z$ and then insert a new vertex $v$ into $F$ by adding edges $v x, v y, v z$. Thus we can start with outer triangle, insert the vertex labelled 2 , insert the vertices labelled 3 into 3 of the faces and so on. We refer to these insertions as operations $2,3,4$, and 5 .

$T_{1}$ is the first in a sequence $T_{1}, T_{2}, \ldots, T_{k}, \ldots$ Construct $T_{2}$ from $T_{1}$ by 'inserting' a copy of $T_{1}$ into each of the 6 "special" faces labelled $3,4,5$. After insertion the vertices inside each special face are numbered as they are in $T_{1}$ and so $T_{2}$ has 36 special faces. In general $T_{k}$ is obtained from $T_{k-1}$ by inserting a copy of $T_{1}$ into each special face and numbering the vertices as above. $T_{k}$ has the following statistics:

(i) $6^{k}$ special faces;

(ii) $t_{k}=\frac{1}{5}\left(16 \cdot 6^{k}-1\right)$ vertices;

(iii) maximum degree 18.

(We obtain (ii) from the recurrence $t_{k}=t_{k-1}+6^{k-1} \cdot 16$.)

Now define $T_{k, i}, i=0,1,2,3,4$ as follows: $T_{k, 0}=T_{k}$ and $T_{k, i}$ is obtained from $T_{k, i-1}$ by applying Operation $i+1$ to those subgraphs contained in each of what was a special face of $T_{k}$. Thus $T_{k, 4}=T_{k+1}$. It is convenient to let $T_{0}$ denote a triangle.

Suppose now that $p=\frac{c(\log n)^{1 / 3}}{n^{1 / 3}}$ where $c=100$. Let $p_{1}$ satisfy $1-\left(1-p_{1}\right)^{10}=$ $p$ so that $p_{1}>p / 10$. We can assume that $G_{n, p}$ is the union of 10 independent copies of $G_{n, p_{1}}$. Let $E_{0}, E_{1}, E_{2}, \ldots, E_{9}$ denote the edge sets of these copies. 


$$
\text { Let } k_{0}=\max \left\{k: 2 t_{k}-4 \leq \frac{1}{2} n\right\}=\left\lfloor\log _{6}\left(\frac{1}{16}\left(\frac{5 n}{4}+11\right)\right)\right\rfloor \text {. }
$$

We try to construct a spanning maximal planar subgraph of $G_{n, p}$ as follows: we will fill in the details of each step of the construction later.

A: construct $T_{0}$ using edges in $E_{0}$;

B: for $k=0$ to $k_{0}-1$ do for $j=1$ to $4 \quad$ do construct a copy of $T_{k, j}$ from the copy of $T_{k, j-1}$ via operation $j$ and using only edges from $E_{r}, r=((k+1) j \bmod 8)+1$

C: augment the copy of $T_{k_{0}}$ to a spanning triangulation by vertex insertion using edges from $E_{9}$ only.

We must now show that we can complete the construction above with probability $1-o(1)$.

A: $G_{n, \frac{\omega}{n}}$ has a triangle with high probability if $w=w(n) \rightarrow \infty$. Since $n p_{1} \rightarrow \infty$ we can be sure that $A$ succeeds with probability $1-o(1)$.

B: the process of constructing $T_{k, j}$ from $T_{k, j-1}$ involves trying to insert a vertex into each of at most $\frac{1}{4} n$ triangles. Suppose that the vertices outside of our copy of $T_{k, j-1}$ comprise $V_{1}(k, j)$ and the vertices of the triangles into which we are trying to insert vertices from $V_{1}(k, j)$ comprise $V_{2}(k, j) \subseteq V_{1}(k-1, j)$. We are examining edges from $E_{r}$. The previous time we used edges from $E_{r}$, the vertices in $V_{2}(k, j)$ were outside of the then current triangulation $T_{k-2, j-1}$ and so the $E_{r}$ edges between $V_{1}(k, j)$ and $V_{2}(k, j)$ are unconditioned by the history of the construction to this point.

To show that $T_{k, j}$ can almost always be constructed from $T_{k, j-1}$ we define a bipartite graph $B P(k, j)$ with vertex partition $V_{1}(k, j)$ and $S(k, j)=\{$ faces $F$ of $T_{k, j-1}$ into which a new vertex is inserted in the creation of $\left.T_{k, j}\right\} . B P(k, j)$ has an edge $v F$ whenever $v \in V_{1}(k, j)$ is adjacent in $G_{r}=\left([n], E_{r}\right)$ to all vertices of $F \in S(k, j)$. Note that $P\left(v F \in E_{r}\right)=p_{1}^{3}$ but that these edges do not appear independently.

To complete the analysis of $B$, we need only prove that

(1) $P(B P(k, j)$ contains a matching of size $|S(k, j)|)=1-o\left((\log n)^{-1}\right)$.

Because the edges of $B P(k, j)$ do not appear independently we again resort to the trick of partitioning the edge set. Let $E_{r}=\bigcup_{i=1}^{7} E_{r, i}$ where the edges of $E_{r, i}$ are chosen independently with probability $p_{2}, 1-\left(1-p_{2}\right)^{7}=p_{1}, p_{2} \geq \frac{p_{1}}{7}$. Consider the graph $\Gamma(k, j)$ which has vertex set $S(k, j)$ and an edge $F_{1} F_{2}$, where $F_{1}$, $F_{2} \in S(k, j)$, whenever $F_{1}$ and $F_{2}$ share a vertex in $T(k, j-1)$. It is not hard to see that the maximum vertex degree in $\Gamma(k, j)$ is at most 7 (when $j=3$ and accounting for special faces sharing a vertex.) It is therefore possible to color these triangles using only 7 colors so that triangles of the same color are vertex 
disjoint. Let us now decompose $B P(k, j)$ as $\bigcup_{i=1} B P(k, j, i)$ where $B P(k, j, i)$ has the $i$-colored triangles denoted $S(k, j, i) \subseteq S(k, j)$, all of $V_{1}(k, j)$ and an edge $v F$ if $v$ is adjacent to all vertices of $F$ via edges of color $E_{r, i}$. Edges in $B P(k, j, i)$ now appear independently with probability $p_{2}^{3}>p^{3} / 70^{3}$. We can now use the result of Erdös and Rényi [2] (see also [1, pp. 155-159]) concerning the threshold for a perfect matching in a random bipartite graph. Actually, we only need a matching from $S(k, j, i)$ to $V_{1}(k, j)$.

By choice of $k_{n},\left|V_{1}(k, j+1)\right| \geq \frac{n}{2}$ always and $\frac{p^{3}}{70^{3}} \cdot \frac{n}{2}>\log n$, so we can first match $S(k, j, i)$ to a subset $V_{1}(k, j, 1)$ of $V_{1}(k, j)$ and then $S(k, j, 2)$ to a subset of $V_{1}(k, j) \backslash V_{1}(k, j, 1)$ and so on, with sufficiently high probability (observe that the dominant failure probability in Erdös and Rényi's result comes from isolated vertices). This completes the analysis of $B$.

C: since the maximum degree in $T_{k_{0}}$ is 18 , each face of $T_{k_{0}}$ shares a vertex with at most 51 other faces. Also $T_{k_{0}}$ has at least $\frac{n}{12}$ faces and so it is possible to find $\frac{n}{612}$ vertex disjoint faces in $T_{k_{0}}$. Furthermore, if a triangulation contains $\alpha$ vertex disjoint faces and a vertex is inserted into one of these faces, then the new triangulation has at least $\alpha$ vertex disjoint faces.

Let $v_{1}, v_{2}, \ldots, v_{m}, m<n$ be an enumeration of the vertices outside of $T_{k_{0}}$. We will try to insert $v_{i}, i=1,2, \ldots, m$ sequentially into the current triangulation using edges in $E_{9}$ only. Since there are always at least $\frac{n}{612}$ vertex disjoint faces available, we have

$$
\begin{aligned}
P\left(\exists i: v_{i} \text { cannot be inserted }\right) & \leq n\left(1-p_{1}^{3}\right)^{n / 612} \\
& <n^{-1 / 2}
\end{aligned}
$$

and this shows that we can complete the construction with high probability and completes the proof of Theorem 1 .

\section{PROOF OF THEOREM 2}

The proof of part (a) is similar that of part (a) of Theorem 1 . Let $\sigma_{n}$ be the number of maximal outerplanar graphs with $n$ labelled vertices. Then

$$
P\left(G_{n, p} \in \mathscr{B}\right) \leq \mathscr{O}_{n} p^{2 n-3} .
$$

But it is known (e.g., Lovász [3, Problem 39 of Ch. 1]) that

$$
\sigma_{n} \leq \frac{(n-2) !}{2}\left(\begin{array}{c}
2 n-4 \\
n-2
\end{array}\right)<\left(\frac{4 n}{e}\right)^{n-2}
$$

and so if $p=(e / 4 n)^{1 / 2}$, then

$$
P\left(G_{n, p} \in \mathscr{B}\right) \leq \frac{c^{2 n-3}}{n^{n-3 / 2}} \mathscr{O}_{n}<n^{-1 / 2}
$$

and this proves part (a). 
For part (b) we provide a construction which can be shown to work with high probability. We once again assume that $G_{n, p}$ is given as the union of a number of independent copies of $G_{n, p_{1}}$. Here four will suffice, so that $1-\left(1-p_{1}\right)^{4}=p$ and $p_{1} \geq \frac{p}{4}$. Let $E_{0}, E_{1}, E_{2}, E_{3}$ denote the edge sets of these copies.

A: construct a triangle $A_{0}$ using edges of $E_{0}$ only.

B: for $k=0$ to $k_{1}=\left[\log _{2} \frac{n}{6}\right]-1$ do

At this point $A_{k}$ is an outerplanar subgraph of $G_{n, p}$ containing $n_{k}=3 \cdot 2^{k}$ vertices. Let the edges of the outer face of $A_{k}$ be $e_{1}, e_{2}, \ldots, e_{n_{k}}$ where $e_{i}, e_{i+1}$ are adjacent $i=1,2, \ldots, n_{k}-1$. Let $F_{1}=\left\{e_{1}, e_{2}, e_{5}, \ldots\right\}$ and $F_{2}=\left\{e_{2}, e_{4}, e_{6}, \ldots\right\}$ be the odd and even indexed edges, respectively.

for $j=1$ to 2 do

construct the bipartite graph $B P^{\prime}(k, j)$ with vertices $F_{j} \cup\left([n] \backslash V\left(A_{k, j-1}\right)\right)$

[ $A_{k, 0}=A_{k}, A_{k, 1}$ is constructed "during" $j=1$ and $A_{k, 2}=A_{k+1}$.]

There is an edge ve, $v \notin V\left(A_{k, j-1}\right), e \in F_{j}$ whenever $v$ is adjacent to both endpoints of $e$ by edges in $E_{j}$.

If $B P^{\prime}(k, j)$ contains a matching of size $\left|F_{j}\right|$, then we can use this matching to add $\left|F_{j}\right|$ vertices to $A_{k, j-1}$ in an obvious way. See Figure 2.

C: augment $A_{k_{1}}$ to a spanning maximal independent outerplanar graph using edges from $E_{3}$ only.

We must now show that we can complete the construction above with probability $1-o(1)$.

A: as for $A$ in the previous section.

B: the edges of $B P^{\prime}(k, j)$ occur independently with probability $p_{1}^{2} \geq \frac{p^{2}}{16}$. We can apply the result of Erdös and Rényi as before since $\left|V\left(A_{k, j-1}\right)\right| \leq \frac{n}{2}$ by definition of $k_{1}$.

C: let $v_{1}, v_{2}, \ldots, v_{m}$ be an enumeration of $[n] \backslash V\left(A_{k_{1}}\right)$. For $i=1,2, \ldots, m$ we try to find an edge on the outerface of the current triangulation for which both endpoints are adjacent to $v_{i}$ using edges in $E_{3}$. Since $V\left(A_{k_{1}}\right)$ has at
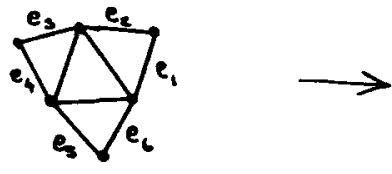

$\hat{A}_{2,1}$

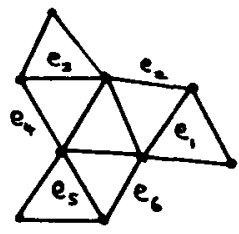

$A_{2,2}$

Fig. 2 
least $\frac{n}{4}$ vertices and the $E_{3}$ edges incident with $v_{i}$ are unconditioned by the previous history we have

$$
\begin{aligned}
P\left(\exists i: v_{i} \text { cannot be added }\right) & \leq n\left(1-p_{1}^{2}\right)^{n / 4} \\
& =o(1) \quad \text { for } c>8 \sqrt{2}
\end{aligned}
$$

This completes the proof of Theorem 2 .

\section{FINAL REMARKS}

The reader will observe that the constants in parts (b) of the theorems can easily be reduced, but that it is not clear how to increase those in parts (a).

The main question left open by this paper is the whereabouts of the exact thresholds. One can also ask for the threshold for the existence of spanning planar subgraphs with $\alpha n$ edges, $\alpha>1$. The argument of parts (a) shows that the threshold is at least $n^{-1 / \alpha}$ and it seems likely that the constructive method we have used can be adapted to attack this problem.

\section{REFERENCES}

[1] B. Bollobás, Random Graphs, Academic Press, Orlando, FL, 1985, xvi + 447pp.

[2] P. Erdös and A. Rényi, On random matrices, Publ. Math. Inst. Hungar. Acad. Sci., 8 , 455-461 (1964).

[3] L. Lovász, Combinatorial Problems and Exercises, North-Holland, Amsterdam, New York, Oxford, 1979, 551 pp.

[4] W. T. Tutte, A census of planar triangulations, Canad. J. Math., 14, 21-38 (1962).

Received February 16, 1990 